vielen Zahlenangaben im Text ermüden etwas; sie hätten vielleicht besser in Tabellen-Form dargeboten werden können.

Nach einer Reihe makabrer Diktaturen erhielt Guatemala mit dem Amtsantritt von Arévalo (1944) erstmals eine Regierung, die nicht ausschließlich die Interessen der Oberschicht vertrat. Unter seinem Nachfolger Arbenz (1951 bis 1954) wurde eine - keineswegs radikale - Landreform durchgeführt, die auch die ungenutzten Ländereien der United Fruit Company trafen. Die engen kommerziellen Verbindungen der UFCO mit den Brüdern Dulles sowie die von der guatemaltekischen Oberschicht zur Diffamierung benützte antikommunistische Hysterie dieser Jahre führte zu dem bekannten CIA-Putsch.

Unter den folgenden, wieder ganz von der Oligarchie beherrschten Regierungen wurden Zigtausende von Familien von den ihnen zugeteilten Parzellen wieder vertrieben und an ihnen zum Teil blutige Rache geübt. Das Thema der Landreform hatte sich dem Bewußtsein der Massen zu tief eingeprägt, um völlig von der Tagesordnung gestrichen werden zu können. Doch was in der Folgezeit unter diesem Begriff geschah, war noch nicht einmal Flickwerk, sondern Augenwischerei und Betrug. Das Eigentum der Großgrundbesitzer wurde nicht mehr angetastet. Statt dessen improvisierte man Kolonisierungsprojekte im urwaldartigen Tiefland, wo Tausende von Familien mit falschen Versprechungen angelockt und dann in menschenunwürdiger Behausung und unter unbeschreiblichen hygienischen Verhältnissen auf einem praktisch unbebaubaren Boden sich selbst überlassen wurden. Die Staatsfarmen, die für eine Verteilung auch ohne Enteignungen zur Verfügung gestanden hätten, wurden statt dessen unter politische Günstlinge verschleudert. Selbst auf dem Papier sahen die kühnsten "Reform“-Projekte in fünf Jahren nur soviel Begünstigte vor, als unter Arbenz in jedem Monat tatsächlich begünstigt worden waren.

Faktisch zielte die Agrarpolitik seit 1954 darauf ab, die indianischen Massen unter dem Existenzminimum zu halten und so den Nachschub an billigen Arbeitskräften für die Großgrundbesitzer zu sichern. Hunderttausende von Wanderarbeitern müssen während der Erntezeit auf den Latifundien arbeiten, wo sie als vorübergehende Arbeitskräfte ohne Rücksicht auf ihre Gesundheit ausgebeutet werden. Das „finstere Mittelalter" und die Sklavenhalter-Gesellschaften erscheinen dagegen als human, da sie der ausgebeuteten Arbeitskraft wenigstens noch einen stabilen Lebensraum zuwiesen und für die Erhaltung dieser Arbeitskraft sorgten. - Streckenweise liest sich das Buch wie eine danteske Beschreibung einer enthumanisierten Welt. Mutwillig werden Gruppen von Baumwollarbeitern vom Flugzeug aus mit giftigen Insektenmitteln besprüht. Arbeiter werden mit List und Gewalt um ihren Lohn geprellt, der die gesetzliche Mindestsumme von 80 Cents kaum je erreicht. Aufgrund gefälschter Besitztitel werden indianische Gemeinschaften von ihrem jahrhundertealten Gemeindebesitz vertrieben. Militär und Polizei sorgen - notfalls mit Folterungen und Mord - für „Ordnung “*.

Tilman Tönnies Evers

\title{
Hubertus Seifert
}

\section{Die Reparationen Japans}

Ein Beitrag zum Wandel des Reparationsproblems und zur wirtschaftlichen Entwicklung Japans nach 1945

\footnotetext{
* Eine Rezension spanischsprachiger Literatur über Guatemala ist für eines der folgenden Hefte vorgesehen.
} 


\section{Internationale Kooperation}

Aachener Studien zur internationalen technisch-wirtschaftlichen Zusammenarbeit 6. Westdeutscher Verlag, Opladen 1971. 208 S.

\section{Chitoshi Yanaga}

Big Business in Japanese Politics

Yale University Press, New Haven and London 1968. IX, 371 S.

\section{KiYoshi KoJima}

\section{Japan and a Pacific Free Trade Area}

Macmillan, London 1971.195 S.

Die dem Deutschen Reich nach dem ersten Weltkriege auferlegten Reparationen haben zu einer umfangreichen Literatur geführt, deren Thema in erster Linie die ökonomische Problematik dieser neuen Art einer Kriegsentschädigung gewesen ist. Eine Neuauflage erfuhr die Reparationspolitik nach dem zweiten Weltkrieg, und wieder stand man vor ähnlichen Fragen wie 25 Jahre zuvor. Im Gegensatz $\mathrm{zu}$ damals entstand jedoch in Ostasien ein Geflecht von Reparationsbeziehungen, das in seiner reibungslosen Abwicklung völlig neue Aspekte zeigte und das Thema des ersten hier vorzustellenden Buches ist.

Der Autor stellt im ersten Kapitel seiner Arbeit zunächst „Wesen, Begriff und Arten der Reparationsleistungen" dar, wobei er insbesondere dafür eintritt, auch Gebietsverluste zu den Reparationen hinzuzurechnen. Gerade im Falle Japans, dem die übrigen drei Kapitel des Buches gewidmet sind, gehören zu den umfänglichen Gebietsverlusten weite und für die Wirtschaft des Inselstaates wertvolle Meeresflächen, deren Nutzung kaum in einem Geldwert ausgedrückt werden kann. Im Falle Japans zeigt sich aber auch besonders deutlich, daß Reparationen trotz gegenteiliger Beteuerungen kaum moralischen Hintergrund besitzen, sondern Instrument reiner Machtpolitik sind. Als solches verlieren sie ihre Bedeutung und ihren Sinn, wenn die Machtkonstellationen sich ändern, wie dies gerade in Ostasien seit der Errichtung der Volksrepulik China und seit dem Korea-Krieg der Fall gewesen war.

Der Autor stellt sehr deutlich die inneren Widersprüche der „klassischen“ Reparationspolitik dar, die in Japan schon frühzeitig wiederholte Revisionen nötig machte. Während eine wirtschaftliche Stabilität Japans zunächst für die Voraussetzung der politischen Stabilität in Ostasien angesehen wurde, betrachtete man doch bald gerade ein schwaches Japan als die größte Gefahr im ostasiatischen Raum. Die USA förderten deshalb den wirtschaftlichen Aufbau und forcierten nicht länger eine Politik der Schwächung ihres ehemaligen Gegners. Nach dem Friedensvertrag von San Francisco (1951) hat Japan dann überhaupt nur noch mit vieren seiner ehemaligen Feinde Reparationsabkommen geschlossen, nämlich mit Birma, Indonesien, den Philippien und Süd-Vietnam.

Wesentlich für die Durchführbarkeit dieser Abkommen war die Tatsache, daß sie in wenn auch manchmal schwieriger, doch letzten Endes in jeweils gegenseitiger Übereinstimmung abgeschlossenen bilateralen Verhandlungen getroffen wurden, bei denen sich die wirtschaftliche Vernunft durchsetzen konnte. Was ferner die reibungslose Durchführung der Abkommen erleichterte, war das Entwicklungsgefälle zwischen Japan und den Empfängerländern, das eigentlich die Reparationen in Entwicklungshilfeleistungen verwandelte. Die Sachlieferungen Japans - zum größten Teil Kapitalgüter - trafen nicht auf die Konkurrenz ähnlicher Industrien in den Empfängerländern, was sich in Reparationsbeziehungen zwischen Industriestaaten immer wieder als besonderes Problem erwies. Und die Empfängerländer in Südostasien waren an den japanischen Reparationslieferungen schon deshalb interessiert, weil diese ihnen eine eigene Industrialisierung und damit einen Schutz gegen einen erneuten japanischen Einfluß zu gewähren schienen. 
$\mathrm{Daß}$ es vor allem in Indonesien und den Philippinen dennoch nicht gelungen ist, den erneuten japanischen Einfluß zurückzuhalten, und daß die ökonomische Entwicklung in Birma und Süd-Vietnam die von Japan empfangenen Anstöße nicht zu nutzen vermochte, ist gewiß nicht die Schuld Japans, sondern ist in der unglücklichen politischen Entwicklung Südostasiens zu sehen. Aber gerade die ökonomische Aktivität Japans, die ihren Anstoß in den Reparationen hatte, trifft auf zunehmende Kritik, so daß man dem Autor wohl nicht zustimmen kann, wenn er von einer "sich immer mehr anbahnenden Harmonie der Interessen“ (S. 159) spricht und die von Japan durchgeführte Erschließung der südostasiatischen Länder als „unverdächtig“ (S. 167) empfindet.

Japan ist sich seiner problematischen Lage hier allerdings durchaus bewußt, und es ist zu hoffen, daß bei Beibehaltung politisch stabiler Verhältnisse vor allem in Indonesien als dem wichtigsten Lande Südostasiens doch eine Kooperation zustande kommt, die von einem vom Autor beschworenen Solidaritätsgefühl der Völker Asiens getragen wird.

Bei der Untersuchung der Auswirkungen der Reparationen auf die Empfängerländer und auf Japan muß man wohl im Falle Japans ein wenig zur Zurückhaltung raten. So erheblich, wie der Autor meint, kann die positive Wirkung der Reparationen auf den japanischen Außenhandel angesichts der in Tabelle 18 ausgewiesenen Proportionen sicherlich nicht gewesen sein. Bedeutsamer ist eben die indirekte Wirkung des Aufbaus neuer und insbesondere langfristiger Handelsbeziehungen. Und wenn der Verfasser die quantitative Bedeutung Südostasiens für die japanische Außenwirtschaft jener der USA gleichsetzt (S. $161 \mathrm{f}$.), so ist er in die Falle der japanischen Außenhandelsstatistik gegangen, die nämlich Länder wie Hong Kong, Indien, Pakistan, Süd-Korea, Taiwan u. a. mit zu Südostasien zählt. Die südostasiatischen Länder im engeren Sinne - der Raum zwischen Birma und den Philippinen - vereinigt nicht 30\%, sondern nur etwa $10 \%$ des japanischen Außenhandelsvolumens auf sich. Dies ist jedoch immer noch genug, um der japanischen Außenwirtschaft die besondere Pflege dieser Länder angelegen sein zu lassen. Ohne die Reparationen Japans hätten sich die heutigen und vielleicht inzwischen sich eher zum Vorteil Japans auswirkenden Handelsbeziehungen sicherlich nicht entwickeln können.

Trotz der wenigen genannten und unerheblichen Einwände hat der Verfasser eine sehr lesenswerte und übersichtliche Studie vorgelegt, die ein notwendiger Bestandteil jeder Literaturauswahl zur Außenwirtschaft Japans sein sollte. Es versteht sich von selbst, daß der Autor als Außenstehender nur die äußeren Erscheinungen seines Themas abhandeln konnte. Um einen Blick hinter die Kulissen der japanischen Politik zu tun, muß man wohl mindestens Japaner sein. Und tatsächlich vermag uns der zweite hier zu behandelnde Autor eine Fülle ergänzender Informationen zu liefern, vor allem, wenn er in seinem Kapitel über die Reparationspolitik darstellt, daß es führende und einflußreiche Geschäftsleute waren, die in den genannten südostasiatischen Ländern die Reparationsverhandlungen für die japanische Regierung geführt haben. Dies ist als Hauptgrund dafür anzusehen, daß diese Abkommen ökonomisch realistisch waren. Für die japanische Wirtschaft waren die Reparationen die „Mitgift“ (S. 204) für spätere glückliche Verbindungen, die ihren Beitrag zum Wirtschaftsaufschwung Japans geleistet haben.

Die enge Verknüpfung zwischen „Big Business" und der Regierung in Japan, die in den Reparationsverhandlungen zum Ausdruck gekommen ist, bildet das Thema des Buches von Yanaga, der als weitere Beispiele die Kartell-Gesetzgebung, die Kernenergiepolitik und den Aufbau der engen Verbindungen zu den USA eingehend darstellt.

Wenn Yanaga vor allem in den ersten fünf Kapiteln seines Buches das Zusammenspiel der großen Wirtschaftsverbände und der Regierungsstellen darstellt, so daß es unmöglich wird, "to determine exactly how and by whom the country is 
governed“ (S. 26); wenn er den verstorbenen Ministerpräsidenten Ikeda zitiert, der die Regierung als den Kapitän, die Wirtschaftsverbände als den Kompaß des Schiffes bezeichnet hat (S. 34); wenn er feststellt, daß das Regierungsgeschäft für die Wirtschaft viel zu wichtig sei, als daß man es Berufspolitikern und Bürokraten überlassen sollte (S. 71); wenn wir erfahren, wie von Yoshida bis Sato japanische Ministerpräsidenten von der Wirtschaft „aufgebaut" oder zur Resignation gezwungen werden (S. $128 \mathrm{ff}$.); und wenn wir andererseits sehen, welche Karrieremöglichkeiten die japanische Wirtschaft ausgeschiedenen Staatsdienern bietet (S. $107 \mathrm{ff}$.), dann muß man dennoch fragen, wieweit hier ein typisch japanisches Phänomen vorliegt. Es unterliegt keinem $Z$ weifel, daß die Interdependenz zwischen Staat und Wirtschaft in Japan, die es letztlich auch ermöglicht hat, daß in einer liberalen Wirtschaft der Begriff der Planung nicht tabu war, sondern sehr wesentlich den Fortschritt vorangetrieben hat (im Gegensatz zur Planungsallergie der Ära Erhard in der Bundesrepublik Deutschland), eine Grundbedingung für das phänomenale Wirtschaftswachstum des ostasiatischen Inselreiches gewesen ist. Ebenso ist es nur innerhalb der japanischen Gesellschaft auf Grund traditioneller Kommunikationsformen möglich gewesen, die engen Kontakte zwischen Wirtschaftsführern und hohen Beamten der Exekutive aufzubauen, ohne Aufsehen in der Offentlichkeit zu erregen. Denn wo sonst spielen Teehauspolitik, familiäre Verbindungen (Keibatsu), Schul- und Universitätsjahrgänge und Landsmannschaften mit den resultierenden gegenseitigen Verpflichtungen eine solche Rolle wie in Japan, wo sie konsequent für die Steigerung des gegenseitigen Wohles genutzt werden? In diesem Zusammenhang sind die Namenslisten, die Yanaga wiedergibt, eine geradezu faszinierende Lektüre. Wenn aber diese traditionellen Elemente der japanischen Gesellschaft eine Beschleunigung der wirtschaftlichen Entwicklung bewirkt haben, so deshalb, weil gerade sie es sind, die schon frühzeitig die Bedingungen geschaffen haben, die im Westen erst heute als die langsam entstehenden Voraussetzungen für das Funktionieren der post-kapitalistischen Gesellschaft erkannt werden, die von ewiggestrigen Orthodoxen noch mit dem verrosteten Handwerkzeug des Marxismus zu deuten versucht wird. In diesem Sinne sei behauptet, daß Yanagas Buch in gewisser Weise nur eine Konkretisierung einer allgemeinen Studie über Japan ist, in der Japan selbst gar nicht genannt wird: Galbraith, The New Industrial State.

In seiner Arbeit über die moderne Industriegesellschaft hat Galbraith genau die Bedingungen aufgezeigt, die Yanaga im Beispiel Japans beschreibt. Wenn Galbraith sagt, daß in der modernen Industriegesellschaft die frühere Trennlinie zwischen Regierung und Privatunternehmen "undeutlich oder gar imaginär" wird, wenn jede Organisation „allmählich die Ziele der anderen“ übernimmt und wenn „infolgedessen jede Organisation eine Fortsetzung der vorherigen" ist*, so trifft dies genau auf Japan zu, wie Yanaga es uns beschreibt.

Ist deshalb Japan eine „unvollendete“ Demokratie, als die sie der Verfasser in seinem Schlußartikel bezeichnet? Oder hat Japan schon unmerklich den Schritt in eine Gesellschaft getan, die mit überkommenen Maßstäben nicht mehr zu messen ist und die uns die eigentlichen Probleme unserer alltäglichen Politik beschert? Yanagas Buch bietet eine Fülle von Anschauungsmaterial für alle, denen diese Frage mehr bedeutet, als eine Modeströmung unserer Tage.

Wenn wir in dieser Betrachtung neuerer Japan-Literatur von der Verbindung Japans zu Südostasien ausgingen und dabei auf die oftmals mißverstandene Bedeutung dieser Region für die japanische Außenwirtschaft stießen, so ist das dritte hier betrachtete Buch geeignet, diese Bedeutung in das rechte Licht zu rücken.

Kojima geht davon aus, daß der Handel Japans mit den südostasiatischen Ent-

* John Kenneth Galbraith, Die moderne Industriegesellschaft. Knaur Taschenbuch 219, München 1971, S. 299. 
wicklungsländern rückläufig ist und sich mehr und mehr den entwickelten Staaten des pazifischen Raumes zuwendet. Er weist auf die auch anderwärts zu beobachtende Erscheinung hin, daß Industriestaaten ihren Rohstoffbedarf in vermehrtem Maß wieder in entwickelten Staaten zu befriedigen suchen, da diese in der Lage sind, bessere Qualität billiger uund pünktlicher anzubieten. Eine Ausweitung des Handels, die Kojima für Japan als erstrebenswert und notwendig annimmt, und eine zur Förderung dieser Ausweitung eventuell notwendige Spezialisierung mit dem Ziel der verbilligten Massenproduktion für einen größeren als den nationalen Markt gelingt nun um so besser, je ähnlicher die Produktions- und Konsumstruktur der Handelspartner beschaffen sind (S. 66). Da jedoch die Intensität des japanischen Handels mit Westeuropa die geringste im Vergleich zwischen allen möglichen Partnerregionen ist, kommt eine Erweiterung des Handels nur gegenüber den entwickelten Pazifikstaaten in Betracht. Dieses sind die USA, Canada, Australien und Neuseeland. Und Japan sieht sich als Partner dieser Länder, unter denen nach Kojima möglicherweise die USA um so eher zu einer Hinwendung zum Pazifik bereit sein werden, als Europa mit der Vergrößerung der EWG innenbezogener werden wird.

Aus dieser Überlegung und aus den vom Autor mit wirtschaftstheoretischer Argumentation vorgetragenen Vorteilen engerer ökonomischer Zusammenarbeit und insbesondere des Abbaus von Handelsschranken ergibt sich der Vorschlag zur Errichtung einer Pazifischen Freihandelszone (Pacific Free Trade Area $=$ PAFTA). Mit reichhaltigem statistischem Material weist der Autor detailliert den Vorteil nach, den eine solche PAFTA für jedes der fünf beteiligten Länder besitzen wird.

Bei dieser Betrachtung übersieht der Autor jedoch einerseits nicht, daß die Staaten des asiatischen Festlandes, insbesondere China, ebenfalls Pazifikstaaten sind. Er ist der Überzeugung, daß die wichtigsten Rohstoffquellen und die aussichtsreichsten Märkte nicht auf dem asiatischen Festlande, sondern im Bereiche der von ihm definierten PAFTA liegen. Andererseits sieht Kojima durchaus die Gefahr für die Entwicklungsländer Asiens, die sich bei engerer Zusammenarbeit der PAFTAStaaten einem "Club der Reichen" gegenübersehen müßten. Er schlägt deshalb ein System der Entwicklungshilfe und der Außenhandelspräferenzen für diese Länder vor, wobei er davon ausgeht, daß erst die Bildung der PAFTA die entwickelten Pazifik-Staaten in die Lage versetzen wird, eine größere Entwicklungshilfe zu leisten und bei sich selbst die strukturellen Veränderungen vorzunehmen, die nötig sind, um die künftig zu erwartenden steigenden Exporte der Entwicklungsländer absorbieren zu können.

Die damit geschaffene Multilateralität der Entwicklungshilfe - so könnte man Kojima ergänzen - würde dann auch endlich Japan von dem Verdacht reinwaschen, daß es über dieses Instrument versuche, seinen alten Traum von der "Großostasiatischen Wohlstandsphäre" nunmehr mit friedlichen Mitteln zu realisieren. In diesem Sinne könnte der von Kojima aufgezeigte Weg auch die Hoffnung für ein sich weiter entwickelndes und prosperierendes Ostasien im Sinne des Ausblicks unseres ersten hier betrachteten Autors sein.

Der Leser allerdings, der die Spannungen aufmerksam registriert hat, die mit der Verkündung der neuen amerikanischen Handelspolitik im Sommer 1971 zwischen Japan und den USA aufgebrochen sind, möchte an der baldigen Realisationsmöglichkeit der PAFTA zweifeln. Tatsächlich ist Kojima nach dem Erscheinen seines Buches inzwischen auch bereit, eine Kern-PAFTA von Japan, Australien, Canada und Neuseeland zu akzeptieren, ehe die USA bereit sein könnten, sich an einem solchen Gebilde zu beteiligen. Gewiß wäre das nur ein unvollständiger Ersatz; aber dennoch sollte man jede regionale Zusammenarbeit zur Förderung des Welthandels begrüßen, sofern solche Zusammenschlüsse nicht gleichzeitig zu 
Abschließungen gegenüber der Außenwelt führen, die durch eine enge Kooperation zwischen den sich formierenden Handelsblöcken - vielleicht gerade in der Frage der Entwicklungshilfe - bekämpft werden müßten.

Bernhard Großmann

\section{JOHN BADGLeY}

\section{Asian Development. Problems and Prognosis}

The Free Press, New York, London 1971, XIV, 210 Seiten

An den Anfang seines Buches, das ein Buch über Asien sein soll, aber allenfalls eines über 13 südostasiatische Länder ist, stellt der Politologe Badgley die Worte eines westlichen Poeten (Goethe) und eines östlichen Politikers (Soedjatmoko), wobei letzterer u. a. sagt: "... one of the greatest problems they (the people in Southeast Asia) are facing is the apparent lack of relationship between problems presented by the external view of the development process and the way the interior view presents them." Sein Buch versteht Badgley als (partielle) Antwort hierauf, als interpretative Evaluierung des politischen Wandels („interor view“) den südostasiatischen Ländern und dessen Implikationen für den Politiker innerhalb wie außerhalb dieser Länderecke.

In einem ersten Teil werden das bisherige und zukünftige asiatische Milieu analysiert und prognostiziert, in einem zweiten Teil die bisherige und zukünftig mögliche oder notwendige Entwicklungs- und Sicherheitspolitik. Einleitend geht der Autor mit der Meinung ins Gericht, Wandel in den asiatischen Ländern durch amerikanische Aktionen herbeiführen oder lenken zu können; Vietnam sieht er als Katalysator allgemeiner (?) Erkenntnis westlicher politischer Impotenz. Zugleich taucht hier das "Leitmotiv“ des Buches auf, die Infragestellung (a) der These vom nation-state als bester Strategie für gelenkten (wirtschaftlichen, sozialen und politischen) Wandel und der Gleichsetzung von Zentralisierung mit Entwicklung und (b) der (eindimensionalen) Überbetonung des supranationalen regionalism - beide zusammen die Grundlagen bisheriger amerikanischer Asienpolitik, von der Annahme einer chinesischen Invasions- oder Subversionsgefahr bestärkt („. . . the US government still believes that, without the strength of numbers, the states of Southeast Asia will be coerced into subservience by their giant neighbors") (S. 192). Badg!ey hält es als iilustrativ für das westliche Verständnis der asiatischen Situation, daß selbst Autoren wie Gunnar Myrdal (Asian Drama), Theodore Geiger (The Conflicted Relationship) und Lucian Pye (Aspects of Political Development) die Möglichkeit übersehen, daß nation-building selbst ein entscheidendes Hindernis für Entwicklung sein kann.

Dagegen konstatiert er für die (südost)asiatischen Länder ein zunehmend divergierendes Lokalbewußtsein, einen stärker werdenden Hang zur lokal-regionalen Autonomie, eine wachsende Segregation zwischen Elite bzw. Bürokratie und der großen Masse des Volkes, speziell der Bauernschaft, eine stärker werdende Isolierug der meisten Zentralregierungen von der Basis im Falle ernstlicher Attacken von oppositioneller Seite und einen allgemeiner werdenden Mangel an Autorität im zivilen Bereich. Diese Trends sind für ihn Anzeichen dafür, daß der Prozeß des politischen Wandels in den (südost)asiatischen Ländern sich weiter verlangsamen werde. Um den langfristigen Interessen der zwei Milliarden Asiaten von heute (und der vier Milliarden in 25 Jahren) zu dienen, sei daher die Frage zu überdenken, wo Souveränität und Autorität innerhalb der staatlichen Gebilde zu lokalisieren 